\title{
Procesos de enseñanza-aprendizaje en escuelas comunitarias e indígenas del sureste mexicano
}

Teaching-learning in community and indigenous schools in southeastern Mexico María Amalia Gracia y Jorge E. Horbath

\section{(2) OpenEdition \\ 12 Journals}

Edición electrónica

URL: http://journals.openedition.org/aa/3979

DOI: 10.4000/aa.3979

ISSN: 2357-738X

Editor

Programa de Pós-Graduação em Antropologia Social (UnB)

\section{Edición impresa}

Fecha de publicación: 1 diciembre 2019

Paginación: 205-226

ISSN: 0102-4302

\section{Referencia electrónica}

María Amalia Gracia y Jorge E. Horbath, «Procesos de enseñanza-aprendizaje en escuelas

comunitarias e indígenas del sureste mexicano», Anuário Antropológico [En línea], v.44 n.2 | 2019,

Publicado el 03 diciembre 2019, consultado el 27 abril 2021. URL: http://journals.openedition.org/aa/ 3979 ; DOI: https://doi.org/10.4000/aa.3979

\section{(c) (i) $\odot$}

Anuário Antropológico is licensed under a Creative Commons Atribuição-Uso Não-Comercial-Proibição de realização de Obras Derivadas 4.0 International. 


\title{
Procesos de enseñanza-aprendizaje en escuelas comunitarias e indígenas del sureste mexicano
}

\author{
Teaching-learning in community and \\ indigenous schools in southeastern Mexico
}

María Amalia Gracia El Colegio de la Frontera Sur (ECOSUR), Unidad Chetumal - México

Jorge E. Horbath El Colegio de la Frontera Sur (ECOSUR), Unidad Chetumal - México

\section{El derecho a la educación de calidad como desafío para Estado y sociedad}

La educación como herramienta para que individuos y grupos se aproximen al conocimiento socialmente producido y accedan a nuevas formas y dimensiones de su vida constituye uno de los derechos humanos fundamentales. En México, la Constitución posrevolucionaria de 1917 estableció dicho derecho a partir de la libertad, laicidad (ajena a cualquier doctrina religiosa) y gratuidad (enseñanza primaria de establecimientos oficiales) (Art. 3ro.) educativa.

Aun en los países en los que se reconoce la universalidad del derecho a la educación, su extensión hacia grupos minoritarios o marginados se ha ido dando históricamente de manera gradual. En México la universalidad se dirigió, principalmente, a la cobertura poblacional y territorial del servicio: inicialmente se apuntó a la educación primaria (1917), posteriormente a la secundaria (1933) y hace quince años a la preescolar (2002). De esta manera, actualmente, la educación básica obligatoria está conformada por tres grados del nivel preescolar, seis grados del nivel primario y tres grados del secundario.

Junto a la progresión en la ampliación de la cobertura escolar, la calidad de la educación se ha posicionado como parte fundamental del derecho a la educación: no alcanza con tener acceso a la escuela sino que la educación promovida en todos los espacios educativos debe ser de calidad para que pueda satisfacer y ajustarse a las necesidades de aprendizaje de los sujetos a los que se dirige; los niños y niñas son los privilegiados por tal derecho, dada su naturaleza de personas protegidas que no tienen posibilidad de valerse por sí mismos.

La Ley General de Educación (LGE) promulgada en 1993 (última reforma publi- 
cada DOF 20-05-2014) observa que "todo individuo tiene derecho a recibir educación de calidad y, por lo tanto, todos los habitantes del país tienen las mismas oportunidades de acceso al sistema educativo nacional" (artículo 2); dicha ley establece las directrices de la educación para población indígena subrayando que la educación básica tendrá las adaptaciones requeridas para responder a las características lingüísticas y culturales de cada uno de los diversos grupos indígenas del país, así como de la población rural dispersa y de los grupos migrantes (Art. 38) que se han ido incrementando cada vez más en el país ante las políticas neoliberales para el campo. El Reglamento Interior de la Secretaría de Educación Pública (SEP) (Art. 16) - órgano que regula la educación en el país - también establece un marco regulatorio para la educación básica dirigida a la población indígena: la modalidad Intercultural y bilingüe instituida a mediados de la década de los noventa del siglo pasado, que presenta avances respecto al anterior modelo bilingüe bicultural en tanto este último concebía a las culturas indígenas como homogéneas y mantenía una visión esencialista sobre lo indígena, al pensar a las comunidades indígenas como rurales, armónicas, solidarias y estáticas (Berteley, 2002); la Dirección General de Educación Indígena (DGEI), surgida en 1978 en un contexto de fuertes reivindicaciones educativas y sociales por parte del movimiento indígena, norma, supervisa y evalúa el sistema educativo intercultural; y la Coordinación General de Educación Intercultural y Bilingüe (CGEIB) se encarga tanto de promover como de evaluar la política educativa en cuanto a su equidad, desarrollo intercultural y participación social.

El Estado tiene un papel central en garantizar la formación y el aprendizaje de la sociedad, aunque ciertos modelos de Estado perpetúan las desigualdades en opciones y oportunidades de los ciudadanos al priorizar ciertas regiones, territorios o grupos sociales. La población indígena ha experimentado condiciones desventajosas en términos educativos pues las políticas de este sector han contribuido en convertir a los indígenas en los grupos y comunidades más desposeídas y vulnerables dentro de la sociedad mexicana. La carencia de opciones educativas o la baja calidad de éstas colocan a los jóvenes indígenas en desventaja con relación al resto de los educandos en el país y, por ende, en franca desventaja de oportunidades al no contar con la formación esperada en los sectores laborales existentes.

Aun si en las zonas rurales de México la educación se ha ido ampliado cada vez más, las condiciones en las que los estudiantes reciben sus clases - infraestructura, educación máxima de los docentes, materiales didácticos y tecnológicos - adoptan el entorno de escasez de insumos y de marginación de las comunidades ¿Cómo 
garantizar el acceso a una educación de calidad en estas condiciones? A partir de este interrogante general, el objetivo del artículo es analizar la evaluación del aprendizaje que realizan los docentes en las escuelas comunitarias e indígenas del sureste mexicano tomando en cuenta el proceso de enseñanza-aprendizaje, a fin de determinar si los docentes involucrados son portadores de la información y la formación necesarias para ajustar el proceso a las necesidades de sus educandos y, por ende, impactar positivamente en el logro educativo.

Partimos del supuesto de que los espacios escolares como la escuela y el aula, los procesos educativos y las formas y mecanismos de evaluación condensan complejos momentos de la vida académica escolar (Monzón, 2015). Para acercarnos a ellos y evaluarlos, es necesario preguntarse ¿por qué/para qué evaluar?, ¿qué evaluar?, ¿cómo evaluar? y, ¿cómo comunicar? (García et al., 2011; López; Hinojósa, 2018). Por otro lado, si buscamos que la evaluación sirva para el mejoramiento de la escuela (Moreno, 2011; Cuevas et al., 2016) requerimos entender el rol que asumen los maestros en dicho proceso (Martínez, 2013; Martínez; Guevara, 2015) e incorporar tanto una evaluación formativa como sumativa. Finalmente, remarcamos que los contextos del proceso de evaluación son sumamente relevantes y particularmente difíciles para los procesos de enseñanza-aprendizaje de calidad cuando los contextos sociales son vulnerables ya que con frecuencia redundan en formas empobrecidas de evaluación (Román, 2003).

\section{Metodología e instrumentos de producción de datos}

La investigación fue principalmente de carácter cualitativa e incluyó entrevistas abiertas, observación participante en las aulas, grupos focales y una encuesta, herramientas diseñadas e instrumentadas para registrar la percepción, los discursos y las acciones y prácticas evaluativas de los actores principales del proceso escolar tales como estudiantes, docentes y funcionarios.

En una primera etapa se realizó análisis documental de los informes estatales de la Secretaría de Educación Pública (SEP) del Consejo Nacional de Fomento Educativo (CONAFE) y de los programas educativos y se entrevistaron cinco funcionarios de la Secretaría de Educación de cada estado. En la segunda etapa, utilizando el Censo de Escuelas, Maestros y Alumnos de Educación Básica y Especial de la SEP se construyó un directorio de cinco escuelas de educación indígena y comunitaria por cada zona rural aledaña a las principales ciudades del sureste mexicano (Campeche, Ciudad de Carmen, Cárdenas, Villahermosa, Chetumal, Cancún, Playa del 
Carmen, Tuxtla Gutiérrez, San Cristóbal de las Casas, Mérida, Tizimín y Valladolid) que permitió, luego de visitas y valoraciones de acceso y viabilidad, seleccionar 13 escuelas, predominantemente comunitarias (ver tabla 1); en ellas se efectuó observación participante de tres clases de un mismo grupo-aula, así como observación de su entorno y condiciones con el objeto de conocer la dinámica escolar, las estrategias docentes y el proceso de enseñanza aprendizaje. Asimismo, se efectuó una encuesta no aleatoria a 134 estudiantes (72 niños y jóvenes varones y 62 mujeres), en su mayoría de secundaria (78\%), para captar el perfil del alumnado, sus expectativas de escolarización, tiempo que destinaban al estudio, estrategias docentes de enseñanza y evaluación, así como características del entorno familiar. La edad de los educandos encuestados varía de acuerdo con el nivel educativo que estaban cursando y, en general, se ajusta a lo que teóricamente corresponde a dicho nivel; ellos viven principalmente en familias de tipo nuclear con padre, madre y hermanos, aunque algunos moran en familias extensas que tienen, en promedio, seis integrantes. Los padres y madres de familia tienen generalmente un nivel de estudios que no supera la educación básica, siendo las madres las que han alcanzado un menor nivel de escolaridad: mientras los padres lograron estudios de secundaria, las madres solo concluyeron la educación primaria.

Esta información se complementó y contrastó con la generada a partir de 11 grupos focales de entre 6 y 10 estudiantes en los que se trabajó la percepción sobre el proceso de evaluación (en total participaron 90 niñas, niños y jóvenes).

\begin{tabular}{|c|c|c|c|}
\hline Ciudad principal & Localidad & Nivel & Grados \\
\hline Cancún & Emiliano Zapata & Secundaria & 1 ero y 2 do \\
\hline Chetumal & San José de la Montaña & Secundaria & 1ero, 2do y 3ero \\
\hline Playa del Carmen & Cristo Rey & Secundaria & 2do y 3ero \\
\hline Mérida & Dzoyaxche & Secundaria & 1ero, 2do y 3ero \\
\hline Tizimín & Kabichen & Secundaria & 1ero, 2do y 3ero \\
\hline Valladolid & Chanyodzonot 2 & Preescolar, Primaria & 1 ero, 2do, 3ero y 6to \\
\hline Campeche & Hobomó & Secundaria & 2do y 3ero \\
\hline Ciudad del Carmen & Juan de la Cabada & Secundaria & 1ero, 2do y 3ero \\
\hline Villa Hermosa & Estanzuela & Secundaria & 1ero, 2do y 3ero \\
\hline Cárdenas & El Carmen & Primaria & 4 to, 5 to y 6 to \\
\hline Tuxtla Gutiérrez & Tuxtla Gutiérrez & Primaria & 5 to y 6 to \\
\hline San Cristóbal & El Pinar & Primaria indígena & 3ero \\
\hline Tapachula & Consejo Agrario Mexicano & Primaria & 5to. \\
\hline
\end{tabular}

Tabla 1. Escuelas de educación indígena y comunitaria del trabajo de campo

Fuente: elaborado a partir de trabajo de campo. 
Finalmente se efectuaron 9 entrevistas a docentes para captar su perfil y los contextos educativos en los que se llevan a cabo el proceso de enseñanza - aprendizaje y su evaluación. Los Líderes Comunitarios entrevistados, 6 varones y 3 mujeres, tenían en promedio 24 años de edad (entre 19 y 30 años) y muy poca antigüedad en el cargo (1.75 años en promedio, desde 1 a 4 años), aunque 7 de ellos tenía experiencia en el sector como asistentes educativos, capacitadores o tutores. Su escolaridad era mayoritariamente de nivel medio superior (6), aunque algunos habían egresados del nivel superior (3). Algo a destacar es que ninguno de ellos era hablante de lengua indígena ni pertenecía a alguna organización vinculada con cuestiones indígenas. Se desempeñaron en el ciclo escolar 2013-2014 en los niveles de primaria (2, uno en la modalidad de aula compartida) y secundaria (7). Todos manejaron enseñanza multigrado, atendiendo simultáneamente sobre todo tres grados (5 casos) y dos grados (3 casos), aunque uno de ellos atendía más de cuatro grados. En promedio, los grupos a su cargo tenían 15 estudiantes, en un rango que iba de 6 a 27. Residían en las comunidades durante la semana y los fines de semana regresan a sus hogares ubicados en otras localidades.

\section{Contexto regional de la educación básica en la región sureste}

A fin de presentar una radiografía de la estructura educativa básica en las entidades federativas de la región sureste, hacemos un balance de centros educativos, docentes y alumnos en cada uno de los niveles, mostrando el peso que tiene la educación indígena y comunitaria en la región y su importancia en la oferta educativa.

\subsection{Los centros educativos}

Según el Censo de Escuelas, Maestros y Alumnos de Educación Básica y Especial, CEMABE 2013, en la región existen 17,668 centros educativos, incluyendo las escuelas construidas, las escuelas móviles y las escuelas que operan en espacios abiertos (sin construcción). De ellos el 93\% son escuelas públicas $(16,413)$ y solo el $7 \%$ son de sostenimiento privado $(1,255)$. Del total el 99\% son escuelas construidas, es decir fijas, de las cuales el 68\% están diseñadas para dar servicio educativo mientras el 29\% son lugares adaptados y el 3\% son escuelas construidas con materiales precarios. El porcentaje de escuelas privadas y escuelas públicas por cada entidad federativa no difiere en mucho en la región; para Campeche el 92.6\% es público, en Chiapas el 95.8\%, en Quintana Roo el 85.8\%, en Tabasco el 95.4\% y en Yucatán $85.6 \%$. Para el total del país, las escuelas públicas cubren el $88.4 \%$, por lo 
que Campeche, Chiapas y Quintana Roo están por encima de lo observado a nivel nacional. El estado de Chiapas tiene 4.1\% de escuelas de educación básica, seguido por Tabasco con el $2.6 \%$ en relación con el total nacional; mientras las otras tres entidades alcanzan el 3\% en conjunto. Todo esto nos habla de una mínima proporción de centros educativos básicos en la región.

\subsection{Los docentes}

El total de docentes de la región sureste (96,337 profesores) representa el 7.8\% del total nacional de docentes: Yucatán (2.1\%) y Tabasco (2.0\%) son los estados que tienen un mayor porcentaje de docentes en relación al total del país, mientras que Campeche solo representa el 0.8\%. En cuanto al lugar de trabajo, el 84.7\% de los docentes laboran en escuelas públicas de la región sureste, porcentaje mayor que el nacional (83\%). Por su parte, el estado de Quintana Roo presenta el mayor número de escuelas privadas con 10996 centros educativos (22.5\%), porcentaje superior a lo observado a nivel regional (15.3\%) y nacional (17\%).

La región sureste concentra al $8 \%$ de los docentes de educación básica del país. De ellos, 22,861 atienden grupos de preescolar, 43,753 de primaria y 28,573 de secundaria, siendo el nivel de primaria el que concentra el mayor porcentaje de profesores (46\%) seguido por el 30\% de profesores de secundaria y el 24\% de preescolar. En el nivel preescolar, las entidades que aglutinan un mayor número de docentes son Chiapas y Tabasco con el $2.8 \%$ y $2.3 \%$ respectivamente del total nacional y $31.2 \%$ y $25.3 \%$ a nivel regional. En el nivel de primaria los estados con mayor número de profesores son Tabasco y Yucatán, representando el 2\% y 1.9\% respectivamente del nivel nacional y el $26.5 \%$ y el $25.5 \%$ respectivamente en términos regionales. Finalmente, en secundaria, son las mismas entidades, Tabasco y Yucatán, las que tienen mayor número de docentes, la primera con el 2.4\% nacional y $26.4 \%$ regional y la segunda con el $7.6 \%$ a nivel país y $31.6 \%$ regional.

Las modalidades de educación indígena y comunitaria tienen importante presencia en la región; observamos que los docentes de dichos servicios presentan una tasa de participación mayor a la indicada a nivel nacional pues los profesores de escuelas indígenas ascienden a 5,220 (que representan el 5.5\% del total), mientras que a nivel nacional los profesores de dicha modalidad alcanzan solamente un 2.6\%. Para el caso de docentes de escuelas comunitarias, esta relación es aún más fuerte: en la región sureste hay 7,734 docentes trabajando en dicha modalidad, lo que representa el $8.1 \%$ que sobrepasa sobradamente el 3\% nacional. El mayor 
porcentaje de docentes de educación indígena de nivel preescolar se concentra en Yucatán (16.35\%), Chiapas (10.11\%) y Tabasco (9.64\%). En lo que respecta al nivel de primaria, Chiapas (16.71\%), Yucatán (14.4\%) y Tabasco (5.54\%) concentran a la mayoría de los docentes que laboran en escuelas indígenas. En cuanto a los docentes de educación comunitaria, observamos que el estado de Chiapas concentra al mayor número, con $41.71 \%$ del total de su plantilla de nivel preescolar, el $25.28 \%$ de nivel primaria y el $10.88 \%$ de secundaria; le sigue el estado de Yucatán con 19.6\% de preescolar, $24.7 \%$ de primaria y $20.7 \%$ de secundaria. El estado de Quintana Roo, en cambio, tiene el menor número de docentes en educación indígena (348 profesores) y en educación comunitaria (270 profesores).

A partir de los datos anteriores podemos hablar de la relación maestro/escuela - en términos del promedio de docentes que laboran en un centro de trabajo. Para el total nacional, esta relación es de 7.1 docentes por escuela, mientras que en la región es de 5.5. Los mejores resultados de este indicador lo obtuvieron las entidades de Quintana Roo con 9.1 profesores por escuela y Yucatán con 8.9, sobrepasando tanto el dato regional como el nacional. En cambio, Campeche con $6.5 \mathrm{y}$ Tabasco con 5.6 solo sobrepasan el nivel regional, mientras que Chiapas queda muy por debajo de ambas escalas con 3 profesores por escuela, lo cual muestra la desventaja que tiene ese estado en cuanto a las modalidades de educación que estamos observando.

\subsection{Los alumnos en la educación básica}

De los 23,562,183 alumnos de educación básica que hay en México, el 7.8\% (1, 844,270 estudiantes) se localiza en la región de estudio. De ellos, el 50.8\% son hombres y $49.2 \%$ mujeres. Tabasco es la entidad con mayor número de alumnos $(551,209)$, a la que le siguen Yucatán $(435,502$ estudiantes) y Chiapas $(374,513)$, representando el 29.9\%, 23.6\% y 20.3\% del total regional respectivamente. En nivel preescolar observamos que existen 4, 305,966 alumnos a nivel nacional (50.5\% hombres y 49.5\% mujeres) y en la región de estudio hay 394, 968 alumnos (199,639 hombres y 195,329 mujeres) que representan el 9.2\% del total nacional. Respecto al total nacional, los estados de Tabasco (121,325 alumnos) y Chiapas (102,392 alumnos) tienen mayor porcentaje de participación, con $2.8 \%$ y $2.4 \%$ respectivamente. A nivel nacional los alumnos de preescolar en escuelas indígenas representan el 6.2\% de la matrícula total, mientras en la región alcanzan el 19.6\%. Para los alumnos de escuelas comunitarias se tiene un 3.7\% nacional y un $21.3 \%$ 
regional. Dentro de las entidades observamos que las escuelas preescolares indígenas tienen un porcentaje importante de la matrícula total en Yucatán (22.6\%), Chiapas $(16.5 \%)$ y Campeche $(12.8 \%)$. En cuanto a las escuelas comunitarias, Chiapas tiene al 24.2\% de sus alumnos en el nivel preescolar y Campeche le sigue con solo el 4.5\%. Los alumnos del nivel primario son casi dos tercios más que los de preescolar y Yucatán es el estado que tiene el mayor número de ellos.

En México hay 13, 151,297 alumnos en el nivel primaria, de los cuales 6, 713,098 son hombres (51\%) y 6, 438,199 mujeres (49\%). El servicio de escuelas indígenas representa un $3.9 \%$ y el comunitario solo el $0.8 \%$. A nivel regional se tiene un total de 1, 004,705 estudiantes (51\% hombres y 49\% mujeres), lo cual representa el $7.6 \%$ del total de alumnos matriculados en el país. Los alumnos de escuelas indígenas primarias en la región sureste son $72,743(14.2 \%)$ y en escuelas comunitarias hay 28,724 (26.3\%). Chiapas sobresale para ambos tipos de servicio educativo: del total de alumnos de primaria, el 22.9\% estudia en escuelas indígenas y el $12.5 \%$ en escuelas comunitarias, lo cual sobrepasa los porcentajes de participación nacionales. De los demás estados, soloYucatán presenta el 5.5\% de alumnos de primaria en escuelas indígenas que es mayor al nacional, pero por debajo de la región. En lo referente al nivel secundario observamos que el total de alumnos a nivel nacional es de 6, 030,943 (50.4\% hombres y 49.6\% mujeres). De ellos sólo el $0.6 \%$ estudia en escuelas comunitarias. Este dato difiere con la situación en la región de estudio, donde la matrícula escolar asciende a 438,238 alumnos, de los cuales el $1.8 \%$ recibe servicio de secundarias comunitarias.

\section{Modelos de educación indígena y comunitaria en México: su peso en la zona de estudio y las principales problemáticas desde la visión de funcionarios del sector}

El modelo de educación intercultural bilingüe forma parte de la oferta educativa hacia la población indígena, aunque no es exclusivo y único para dicha población pues a las escuelas que lo siguen pueden asistir tanto estudiantes indígenas como mestizos. Por su parte, la educación básica comunitaria es un modelo educativo del Consejo Nacional de Fomento Educativo (CONAFE) para la población escolar que habita en las zonas rurales, indígenas, migrantes y de alta marginación y/o rezago social en comunidades dispersas y de poca población. Atiende los niveles de preescolar, primaria y secundaria; a partir de un modelo que involucra a la comunidad y convoca a la colaboración de jóvenes en la impartición de los servicios educati- 
vos “diseña, desarrolla y opera modalidades y programas educativos flexibles, pertinentes, bilingües e interculturales, basados en las necesidades de aprendizaje de los pueblos indígenas, de los habitantes de las pequeñas localidades rurales y de las zonas urbano marginales, así como de los campamentos agrícolas, albergues indígenas y comunidades de origen de población jornalera migrante" (CONAFE, 2014, p. 4). Esta modalidad apoya con útiles escolares, materiales, auxiliares didácticos, mobiliario escolar para los servicios educativos, capacitación para las Asociaciones de Padres de familias y formación de las Figuras Educativas, construcción o rehabilitación de espacios educativos, apoyos económicos para las Asociaciones Promotoras de Educación Comunitaria, para prestadores de Servicio Social Educativo (instructores comunitarios, agentes educativos comunitarios, capacitadores tutores, asistentes educativos, asesores pedagógicos itinerantes y tutores comunitarios de verano) y para beneficiarios de las becas "Acércate a tu Escuela" para continuar con los estudios y para docentes.

De los datos del Censo de Escuelas, Maestros y Alumnos de Educación Básica y Especial (CEMABE) de 2013 (SEP, 2013b) se observa que las modalidades de educación indígena y comunitaria tenían una importante presencia en la región sureste y que los docentes involucrados en dichos servicios presentaban una tasa de participación considerablemente mayor que los del nivel nacional: los profesores de escuelas indígenas eran 5,220, es decir, el 5.5\% del total - mientras que a nivel nacional alcanzan solamente $2.6 \%$ - y los de las escuelas comunitarias eran 7,734 docentes, lo que representaba el 8.1\% - frente al 3\% nacional.

En las escuelas de educación indígena, los municipios de San Cristóbal de las Casas, Tapachula y Valladolid concentraban un mayor número de alumnos. En la región había 26.4 alumnos por profesor, pero en Mérida la relación era mayor con 30.1 alumnos por docente, mientras que en San Cristóbal era de 29.6 alumnos, en Tapachula de 29.2 y en Tuxtla Gutiérrez de 27.1 alumnos por docentes (Tabla 2). 


\begin{tabular}{|c|c|c|c|c|c|c|c|c|c|c|}
\hline \multirow[t]{2}{*}{ Concepto } & \multicolumn{3}{|c|}{ Educación Indígena } & \multicolumn{3}{|c|}{ Educación Comunitaria } & \multicolumn{3}{|c|}{ Resto Educación Básica } & \multirow{2}{*}{$\begin{array}{l}\text { Cober- } \\
\text { tura* }\end{array}$} \\
\hline & Escuelas & Docentes & Alumnos & Escuelas & Docentes & Alumnos & Escuelas & Docentes & Alumnos & \\
\hline \multicolumn{11}{|c|}{ estado de Quintana Roo } \\
\hline $\begin{array}{l}\text { OTHON P. } \\
\text { BLANCO }\end{array}$ & $\overline{0}$ & $\overline{0}$ & 0 & 44 & 43 & 477 & 329 & 2277 & 47121 & 24.5 \\
\hline $\begin{array}{l}\text { BENITO } \\
\text { JUAREZ }\end{array}$ & 0 & 0 & 0 & 19 & 16 & 181 & 570 & 6266 & 132747 & 20.0 \\
\hline SOLIDARIDAD & 0 & 0 & 0 & 5 & 5 & 43 & 133 & 1388 & 32067 & 16.0 \\
\hline \multicolumn{11}{|c|}{ estado de Campeche } \\
\hline CAMPECHE & 11 & 17 & 369 & 15 & 17 & 133 & 301 & 2611 & 52057 & 9.1 \\
\hline $\begin{array}{l}\text { CIUDAD DEL } \\
\text { CARMEN }\end{array}$ & 0 & 0 & 0 & 61 & 56 & 456 & 308 & 2273 & 48930 & 3.3 \\
\hline \multicolumn{11}{|l|}{ Estado de Yucatán } \\
\hline MÉRIDA & 7 & 20 & 553 & 25 & 26 & 255 & 1048 & 9239 & 168192 & 5.2 \\
\hline TIZIMIN & 33 & 79 & 1835 & 67 & 67 & 673 & 85 & 702 & 15914 & 18.0 \\
\hline VALLADOLID & 35 & 136 & 3413 & 36 & 25 & 215 & 75 & 771 & 15049 & 14.2 \\
\hline \multicolumn{11}{|l|}{ estado de Tabasco } \\
\hline CENTRO & 25 & 90 & 1892 & 54 & 56 & 604 & 786 & 5733 & 139301 & -0.9 \\
\hline CARDENAS & 0 & 0 & 0 & 31 & 33 & 395 & 410 & 2447 & 61984 & 19.0 \\
\hline \multicolumn{11}{|l|}{ estado de Chiapas } \\
\hline $\begin{array}{l}\text { SAN CRISTO- } \\
\text { BAL DE LAS } \\
\text { CASAS }\end{array}$ & 77 & 271 & 8275 & 35 & 38 & 321 & 232 & 1618 & 41102 & 10.0 \\
\hline TAPACHULA & 51 & 143 & 3863 & 45 & 61 & 771 & 499 & 2947 & 71312 & 14.4 \\
\hline $\begin{array}{l}\text { TUXTLA } \\
\text { GUTIERREZ }\end{array}$ & 3 & 8 & 238 & 17 & 27 & 317 & 504 & 4596 & 119316 & 9.4 \\
\hline
\end{tabular}

Tabla 2. Educación básica, indígena y comunitaria en los municipios del cinturón urbano de la región sureste de México. Ciclo escolar 2011-2012.

* Base de referencia la de población de 3 a 14 años del Censo de Población de 2010. Fuente: Cálculos propios con base en estadísticas de la Secretaría de Educación Pública de México.

Estos datos muestran una complicada situación para la docencia en escuelas indígenas debido a que los profesores están obligados a atender grupos muy numerosos con los cuales la interacción es más limitada, a la vez que los alumnos no logran recibir una atención personalizada y ajustada a sus necesidades.

El mayor número de escuelas comunitarias en los municipios de estudio está en Tizimín, estado de Yucatán, El Carmen, estado de Campeche, y Centro, estado de Tabasco. En estas escuelas, la proporción alumno/docente es alta en Solidaridad (Quintana Roo) con 16.3 alumnos, Tuxtla Gutiérrez (Chiapas) con 14 alumnos y Tapachula (Chiapas) con 13.6 alumnos por docente, lo que implica que existe un docente atendiendo, simultáneamente, a más de 10 alumnos de distintos grados 
simultáneamente con diferentes objetivos de aprendizaje y planes de estudio (Tabla 2).

Los cursos comunitarios se establecen en comunidades rurales de localidades con menos de 500 habitantes, así como en campamentos agrícolas y albergues. Este es el único modelo educativo en el país que solicita a las familias de los estudiantes apoyar en la edificación y mantenimiento de los centros escolares. En muchos de los casos observados, la instauración de los cursos comunitarios respondió a la solicitud de padres y madres de familia hacia las autoridades municipales, la Secretaría de Educación o el propio CONAFE, quienes se comprometieron a donar el terreno y ofrecer la mano de obra para construir las primeras aulas.

En los municipios de estudio y en términos agregados, la cobertura de la educación básica es significativamente amplia en casi todos ellos, con excepción del municipio de Centro en el estado de Tabasco en el que impacta el efecto del área metropolitana de Villahermosa y su mayor cobertura extendida hacia la población de otros municipios vecinos (Tabla 2).

Las escuelas estudiadas fueron creadas relativamente de forma reciente: desde 1998 a la fecha y cuatro de ellas no tienen más de cinco años de creación. En promedio, en las escuelas secundarias se imparten clases durante poco menos de siete horas (6.85), mientras que las primarias lo hacen durante seis horas, en todos los casos en horario matutino.

Las escuelas constan de un aula en la que se atiende a un grupo multigrado y sólo dos escuelas tienen dos y cuatro aulas; todas ellas presentan ventilación insuficiente y cuentan con algún espacio que funciona como biblioteca (suele ser un estante o mesa). Salvo en una de las escuelas en que los alumnos utilizan los baños de sus casas, todas poseen sanitarios. No tienen área administrativa ni instalaciones deportivas y el mantenimiento de las instalaciones lo hacen los padres de familia y los alumnos, especialmente las madres que se encargan del aseo de los baños.

Los funcionarios entrevistados comentaron que el éxito de los programas de educación comunitaria está dado por su capacidad para llegar a comunidades alejadas que ninguna otra instancia atiende, pero que actualmente no logran cumplir con el perfil de egreso establecido para la educación básica general, por lo cual entre sus metas está "no llegar a ser mejores, pero al menos llegar a ser iguales" (Entrevista a funcionario CONAFE del estado Campeche).

Otro problema reiterado es la situación económica de las comunidades para afrontar el compromiso que asumen en los convenios de colaboración que les otorgan la responsabilidad de proporcionar al instructor el hospedaje y la alimentación. 
En el marco de la revisión del modelo educativo de CONAFE, las autoridades coincidieron en señalar como algo fundamental en dicho proceso el diseño de material específico para atender a la población indígena en sus lenguas pues señalaron que la escasez de material didáctico es una de las principales limitaciones para la implementación de los programas de estudio, especialmente para la población indígena que asiste a las escuelas comunitarias. Peso a lo anterior, no todas las escuelas comunitarias reúnen las características requeridas para aplicar este programa de dotación de libros y biblioteca.

Las herramientas o instrumentos de trabajo con los que actualmente cuentan los Líderes Comunitarios para ejercer tu función como docentes son los manuales del instructor, las guías de trabajo y el manual de evaluación. Desde la visión de los funcionarios, el hecho de que estos jóvenes provengan de fuera de las comunidades influye en el alto grado de deserción que se observa en la educación comunitaria. Para el caso de los estudiantes de secundaria varones, la problemática de la deserción se agudiza porque apoyan diariamente y desde temprano en las actividades agrícolas familiares, aunque en época de cosecha se implementan adecuaciones curriculares para que el proceso de aprendizaje pueda ser más autodidacta y cuente con asesoría personalizada del instructor cuando sea posible.

Los Líderes Comunitarios tienen tutorías mensuales donde presentan sus problemáticas específicas y acreditan conocer los contenidos curriculares básicos; los que se desempeñan en medio indígena teóricamente deben cumplir con el requisito de hablar la lengua indígena del lugar, aunque de acuerdo con los funcionarios tienen una gran dificultad para escribirla (lo cual también se busca atender mediante tutorías mensuales). En la capacitación que se les proporciona se incluyen temas de salud, prevención de enfermedades contagiosas y derechos humanos. Finalmente, pese a la centralidad de su figura, los funcionarios destacan su lejanía con el magisterio.

\section{El proceso de enseñanza-aprendizaje y su evaluación desde las prácticas y percepciones de docentes y estudiantes}

El proceso de enseñanza-aprendizaje suele integrar aportes de diferentes corrientes psicológicas y pedagógicas por lo cual en las prácticas docentes encontraremos tanto principios más conductistas que pretenden cambios de conducta a partir de ciertos condicionamientos así como aspectos vinculados con distintas corrientes del constructivismo que han tenido una mayor acogida contemporáneamente y que enfatizan tanto la participación del aprendiente en dicho proceso como el vínculo 
con el medio ambiente. Desde el constructivismo,

el conocimiento y el comportamiento son el resultado de procesos de construcción subjetiva en los intercambios cotidianos con el medio circundante (...) los procesos educativos preocupados por estimular y orientar el desarrollo pueden concebirse como procesos de comunicación que potencien los intercambios del individuo con el medio físico y psicosocial que rodea al sujeto (Gimeno Sacristán; Pérez Gómez, 1996, p. 45).

La perspectiva del aprendizaje significativo de Ausubel aplicado al caso particular del aprendizaje escolar constituye una aportación sustantiva de la teoría cognoscitiva; para lograr que el aprendizaje sea significativo es necesario que el material posea una coherencia interna y una secuencia lógica entre sus partes, a la vez que dichos contenidos sean comprensibles de acuerdo con la estructura cognitiva del sujeto que aprende. Desde la perspectiva del aprendizaje significativo, el conocimiento está indisolublemente ligado al desarrollo del proceso de aprendizaje dado que "el bagaje ideativo del individuo se enriquece y modifica sucesivamente con cada nueva incorporación” (Gimeno Sacristán \& Pérez Gómez, 1996, p. 48).

Para analizar el proceso de enseñanza-aprendizaje y las actividades de evaluación, diferenciamos entre la evaluación sumativa, que se realiza al finalizar un período lectivo y cuyos reactivos muchas veces son descontextualizados o abstractos pues deben aplicarse de manera universal, y la evaluación formativa, que no solo da cuenta del aprendizaje, sino que permite a los maestros nutrir sus prácticas docentes. En este sentido, el modelo de evaluación formativa está más apegado a la función pedagógica - didáctica de la evaluación y requiere que los objetivos de aprendizaje y los criterios sobre los que se juzgarán los trabajos escolares sean claros y comprensibles de igual forma para maestros y estudiantes; asimismo, demanda definir criterios para evaluar el desempeño de cada estudiante e incluir estrategias de autoevaluación y coevaluación de los alumnos (Esquivel, 2009).

Tanto las entrevistas como las observaciones muestran que la planeación de las sesiones se realiza de acuerdo con los requerimientos establecidos en los manuales. Los docentes analizan los contenidos y se documentan, practican los temas y se ajustan a las características y necesidades de cada uno de los alumnos que forman los grupos para establecer actividades. Sin embargo, si bien los docentes de secundaria tienen acceso a materiales que les proporciona el CONAFE, se pudo observar, a partir de las clases y entrevistas, que no los aplican de la misma manera y 
tampoco dedican el mismo tiempo a cada asignatura ni tampoco a la planificación de las clases. Entre las asignaturas que les resulta más difícil para enseñar mencionaron química, matemáticas e inglés, las cuales, junto con historia, también son difíciles para los estudiantes. Las clases suelen comenzar con un repaso de la sesión anterior; las formas de hacerlo incluyen preguntas directas, aunque también los docentes mencionaron otras formas. El repaso de la clase anterior se realiza para reforzar conocimientos vistos, pero también como preámbulo a un nuevo tema en donde se trata de relacionar lo aprendido con actividades que se realizarán o el tema que se tratará. Las actividades de cierre de sesión pueden ser de diferentes tipos; desde dinámicas de tipo lúdico, a ejercicios más formales que concluyen los temas y promueven la reflexión sobre éstos. Aun si varios docentes indicaron realizar este tipo de actividades, ésta no es una práctica generalizada entre todos los entrevistados. Quienes realizan actividades de cierre no lo hacen diariamente, principalmente porque no siempre les alcanza el tiempo. Las actividades de cierre de bloques son más comunes en las aulas observadas y suelen incluir evaluaciones parciales que formarán parte de la evaluación final del curso. Dichas tareas ofrecen una retroalimentación al docente de los temas vistos y pueden contener repasos de lo que ya se abordó o ser generalizadoras y abarcar varios temas. Entre este tipo de actividades se incluyen evaluación de las unidades de aprendizaje o se hace mención ante el grupo de los logros e indicadores del bloque para confirmar si adquirieron esos conocimientos. En las evaluaciones se generalizan los contenidos abordados dentro del mismo bloque o tema parcial mediante trabajos escritos grupales o individuales, presentaciones orales o exámenes, tanto orales como escritos. Es de destacar que las actividades que se realizan para evaluar no parecen ser tan pertinentes como las de cierre de cada sesión pues no proporcionan tanta retroalimentación del proceso de enseñanza-aprendizaje al tiempo que se advierten vacíos en el modo en que los docentes interpretan los manuales.

Una actividad que los estudiantes refieren como predominante es el leer en voz alta, la cual es valorada por los docentes como algo que les proporciona mayor seguridad a los alumnos y también les asegura que las consignas y actividades se comprenden. Otras actividades sobresalientes de acuerdo con los niños y jóvenes son la escritura de los aprendizajes diarios y la elaboración de esquemas o mapas conceptuales. Exponer trabajos ante el grupo también figura como una de las actividades que suelen realizar de manera cotidiana.

Aun si no utilizan los términos antes mencionados, los docentes afirman que la 
evaluación del proceso de aprendizaje se realiza tanto de manera cuantitativa - en gran medida, semejante a lo que definimos como evaluación sumativa - como cualitativa - que se acerca a lo que definimos como evaluación formativa - e incluye distintos instrumentos como exámenes escritos, carpetas de evidencias, autoevaluación de los estudiantes y tareas, principalmente grupales.

En el modo cuantitativo, el principal instrumento que aplican los docentes son pruebas parciales escritas que realizan cada dos meses, es decir, aplican cinco de estas pruebas en un período escolar. Aun si no tienen un examen final, en el último parcial se incluyen contenidos vistos durante todo el año. Dos aspectos son críticos respectos a las pruebas escritas. En primer lugar, las evaluaciones están redactadas y escritas en español, lo cual pone en desventaja a los estudiantes cuya lengua materna es indígena y constituye una violación a garantizar la educación intercultural. Como relata un docente,

(...) algunos compañeros trabajan en comunidades que son 100\% indígenas y los alumnos tienen dificultad de leer en español y, desafortunadamente, e los exámenes que vienen, es en español todo y para que pueden realizarlos tiene que estar allá el Líder Comunitario asesorándoles (...) (Docente CONAFE, comunidad de Kabichen, Yucatán).

Por otro lado, durante algún tiempo, los asistentes educativos de cada región y/o delegación del CONAFE, diseñaban los reactivos de las pruebas de manera colegiada, inclusive una docente explicó que tenían un espacio para revisar borradores y dar comentarios en reuniones convocadas a nivel regional. Sin embargo, recientemente, la elaboración de las pruebas pasó a tener un carácter nacional: las delegaciones reciben los exámenes que, a su vez, entregan a los docentes para que los apliquen; esto genera discrepancias entre los contenidos evaluados en los exámenes y los impartidos en clases por lo cual muchos docentes se muestran inconformes (para el caso del estado de Tabasco); otros dicen que como estos exámenes solo representan un aparte de la calificación, ellos tratan de compensar este problema con otras actividades, mientras que algunos refirieron que conocen las pruebas de manera anticipada por invitarlos a las reuniones. Aunque de lo anterior se infiere que las realidades son disímiles, la mayoría de los estudiantes perciben que los exámenes se ajustan a los temas vistos en el aula y corresponden con el nivel en el que se encuentran; sin embargo, al preguntar a los estudiantes sobre los aspectos que se pueden evaluar mediante un examen escrito, la mayoría se muestra inseguro y 
tiene, frente a este tipo de pruebas, una actitud de nerviosismo, aun si la mayoría de las veces se prepara para las pruebas. Entre las maneras más comunes de estudio mencionaron el "repaso de los apuntes de clase", la lectura de lo que se vio del tema o la realización de ejercicios hechos en clase.

El modo cualitativo de evaluación incluye aspectos como la participación, la disciplina, la responsabilidad, el compromiso, el trabajo en equipo y la puntualidad, entre otros, y es considerado por algunos de los profesores entrevistados como el enfoque más acertado, pues permite evaluar realmente si el alumno pone en práctica los conocimientos adquiridos.

Una actividad muy importante prevista dentro del modelo educativo en dirección a la evaluación formativa es el diagnóstico con el que inician el año los docentes que constituye un parámetro de las habilidades de cada alumno.

"El diagnóstico se hace normalmente en las vacaciones. Mientras a nosotros nos preparan hay una parte que se llama "prácticas de campo"; a uno lo mandan a la comunidad y en ese tiempo tienes que ir conociendo a tus alumnos (...) Incluso se les puede poner algo de un grado antes, para saber si esos conocimiento del ciclo anterior sí los obtuvieron o no, por ejemplo al que está pasando a tercero se le pone uno de segundo, o el de primero se le pone temas de primaria (...) a uno le dan la indicación de que tienes que ir a la comunidad y levantar como un diagnóstico, si lo quieres hacer a través de un examen escrito o a través de actividades, uno lo prepara" (Docente CONAFE, San José de la Montaña, Quintana Roo).

Este diagnóstico, que como observa el Líder Comunitario de San José de la Montaña, puede tener distintas características según lo crea oportuno el docente, también incluyó en uno de los casos una valoración de las condiciones de la comunidad que incluía aspectos sociales, culturales y de salud, además de actividades económicas de los padres y madres de familia, lo que le dio a ese Líder docente un conocimiento más profundo del entorno en el cual trabajaría, facilitándole el vínculo con sus estudiantes, los padres y madres de éstos y la comunidad. Junto al diagnóstico, los docentes refieren que las participaciones de los estudiantes en las sesiones - que les habilita reconocer de manera más efectiva las habilidades y ritmos de aprendizaje - son las que definen las posibles estrategias que utilizan para avanzar sobre los objetivos de aprendizaje y de adquisición de capacidades.

Los estudiantes destacan que algo que les ayuda y realizan con frecuencia es la revisión de tareas; si bien como resultado de esta actividad el maestro asigna una 
calificación consideramos que esta actividad se constituye un espacio que los docentes emplean para acompañar el proceso de aprendizaje de sus alumnos y solventar a tiempo aspectos que pudieran ser reencaminados, así como poder revisar y aclarar dudas.

En los grupos focales, los alumnos se explayaron sobre las actividades mediante las cuales son evaluados entre las que destacaron exámenes, tareas, preguntas, autoevaluación diaria basada en una ficha, preguntas, proyectos, talleres, investigaciones, participaciones, exposiciones, experimentos. Como se puede observar, el tipo de actividades desarrolladas involucran la participación activa de los alumnos para quienes lo que se busca, sobre todo, es aplicar los conocimientos adquiridos y concentrarse en su aprendizaje al permitirles reconocer sus errores y aciertos y para mejorarlo, aunque ello puede significar su reprobación.

Muy pocos estudiantes advirtieron que la calificación puede ser un medio para mejorar la enseñanza de los maestros y, en general, desconocen el proceso de cálculo de las calificaciones, aunque son conscientes de los aspectos que consideran los maestros para evaluarlos y explicitan sentirse a gusto con el proceso de evaluación en general.

Desde la perspectiva general de los docentes, el propósito de la evaluación se vincula con la verificación del avance de los alumnos y busca conocer su aprendizaje y dificultades. Sin embargo, pese a lo anterior notamos que en muy pocos casos se reflexiona abiertamente sobre la efectividad de las estrategias de enseñanza-aprendizaje, sus ventajas o desventajas o acerca de los temas que requieren mayor dedicación ya sea porque les cuesta a ellos y/o a los estudiantes. Finalmente, un tema poco mencionado en las evaluaciones es la del docente y su proceso de formación/ aprendizaje, aunque la autoevaluación del docente y de sus prácticas de enseñanza, permite buscar explicaciones del éxito o el fracaso del aprendizaje y sirve como punto de partida y complemento para enriquecer, perfeccionar y mejorar las prácticas docentes para fomentar al aprendizaje (Martínez Rizo, 2008).

\section{Conclusiones}

Las modalidades de educación indígena y comunitaria se han diseñado para enfrentar las fuertes desigualdades y rezagos educativos en contextos aislados de las zonas rurales en México y tienen una fuerte presencia en la región de estudio. En los municipios donde se ubican las escuelas indígenas se observó que hay varios en los que los profesores están obligados a atender grupos muy numerosos con los 
cuales la interacción es más limitada, a la vez que los alumnos no logran recibir una atención personalizada ajustada a sus necesidades. Para el caso de la educación comunitaria, hay municipios en los que la relación docente/alumno es alta lo cual confirma la idea ya generalizada de que el subsistema educativo comunitario presenta una situación compleja que determina el que se presenten bajos niveles de aprovechamiento y alta deserción por parte del alumnado.

La educación básica en la zona de estudio tiene un nivel aceptable de disponibilidad o dotación en cuanto a cobertura, es decir hay centros educativos suficientes para satisfacer la demanda. En el caso de las escuelas comunitarias, ellas son espacios que han buscado alcanzar la accesibilidad en el sentido de la no discriminación y/o exclusión a partir de un enfoque educativo que lleva educación formal a las regiones rurales más apartadas. Sin embargo, la pertinencia de los contenidos curriculares respecto a las especificidades culturales, las cualidades profesionales de los docentes y el respeto por los derechos de los pueblos indígenas y su derecho de aprender en sus propias lenguas cuestionan su aceptabilidad en términos educativos. La incorporación de contenidos propuestos por los docentes que conlleven modificaciones de los programas es una de las carencias observadas en el modelo educativo comunitario. En relación a la pertinencia de sus contenidos, los funcionarios destacaron que sólo existe una asignatura estatal que podría responder a las especificidades de cada entidad en la que se revisan contenidos de historia y geografía con la idea de exaltar y reforzar la identidad local y regional, aunque en ella no siempre se recogen los contenidos específicos que debieran ser tratados.

De esta manera, aun si los esfuerzos son enormes, el impacto en términos educativos termina siendo un paliativo de la situación pues no se acerca a los niveles de logro educativo que alcanzan los estudiantes de las escuelas de educación básica en el ámbito urbano. En estos escenarios, los procesos de enseñanza-aprendizaje se ven tensionados entre las formas de transmisión del conocimiento ancestral y la homologación de capacidades para niños y jóvenes a partir de una tarea docente que reproduce información y vigila que llegue y sea asimilada por los estudiantes dentro del aula. Tal dinámica en las escuelas de educación indígena y comunitaria se dificulta más cuando los docentes cuentan con pocas herramientas, incluyendo los déficits en formación y pedagogía, como ocurre en el sureste mexicano.

Desde la perspectiva de los docentes de educación comunitaria es difícil apuntar las características particulares de la evaluación en la educación general puesto que poco conocen sobre este sistema; probablemente varios estudiaron en escuelas for- 
males y pueden tener una referencia de la forma de evaluar, aunque no se corresponda totalmente con la realidad. Algunos se limitaron a hacer recomendaciones generales a la educación comunitaria y especialmente a la dotación de materiales, mayor uso de los libros de texto y el mejoramiento de la infraestructura escolar, recomendaciones que se relacionan con su corta experiencia docente pues, en promedio, ellos no alcanzan los dos años de antigüedad en el cargo y fundamentalmente hacen recomendaciones a sus pares; muchos se muestran inconformes con los exámenes escritos que se presentan cada bimestre dado que evalúan contenidos no vistos en las aulas o porque no contemplan sus observaciones. Algo a resaltar es la importancia que pueden adquirir en el proceso de enseñanza-aprendizaje tanto la autoevaluación como la evaluación grupal, última que prácticamente no se utiliza en las escuelas observadas. La primera motiva a los estudiantes a ser capaces de evaluar su propia práctica y aprendizaje; y la segunda supone que, a partir de la evaluación de los compañeros, los estudiantes no sólo aprendan a realizar valoraciones críticas y constructivas del trabajo de los demás sino también propicia un espacio en el que tanto alumnos como docentes pueden discutir las evaluaciones.

Los aspectos expuestos muestran las limitantes en el proceso de aprendizaje que conectan con las formas de enseñanza en modelos educativos principalmente rurales y distantes de las grandes ciudades. Es en el aula y en la relación docente-alumnos donde se expresan las más fuertes falencias que hasta ahora no han podido ser subsanadas por quienes diseñan la política y los programas de educación dirigidos a comunidades rurales e indígenas. Para superarlas es necesario incluir a las propias comunidades en los diseños curriculares y en las propuestas de evaluación, propiciar el mejoramiento de las capacidades de docentes y supervisores en los procesos formativos y ampliar la dotación en capital físico de los espacios y recursos educativos destinados a los alumnos. Las soluciones no están en eludir la responsabilidad social e histórica cancelando estos programas sino más bien en fortalecerlos para que incidan en el desarrollo social y local de las comunidades donde operan.

Recebido: 30/04/2019

Aprovado: 28/08/2019 
María Amalia Gracia es Profesora-Investigadora Titular del Departamento de Sociedad y Cultura de El Colegio de la Frontera Sur (ECOSUR) Unidad Chetumal. Miembro del Sistema Nacional de Investigadores Nivel II del Consejo Nacional de Ciencia y Tecnología de México (CONACYT). Forma parte del Grupo de Trabajo "Indígenas y espacio urbano" del Consejo Latinoamericano de Ciencias Sociales (CLACSO). Orcid: 0000-0002-1920-5618. Contacto: magracia@ecosur.mx

Jorge E. Horbath es Profesor-Investigador Titular del Departamento de Sociedad y Cultura de El Colegio de la Frontera Sur (ECOSUR) Unidad Chetumal. Miembro del Sistema Nacional de Investigadores Nivel III del Consejo Nacional de Ciencia y Tecnología de México (CONACYT). Coordinador del Grupo de Trabajo "Indígenas y espacio urbano" del Consejo Latinoamericano de Ciencias, Sociales (CLACSO). Orcid: 0000-0002-7849-2207. Contacto: jhorbath@ecosur.mx

\section{Notas}

1. Agradecemos al Consejo Nacional de Ciencia y Tecnología - CONACYT, que financió el proyecto SEP-INEE 2012-1 titulado "Prácticas de evaluación de aprendizaje en el aula en las escuelas de educación indígena y comunitaria en el sureste de México", que sirvió de base para confeccionar el artículo.

\section{Referencias}

BERTELEY, María. 2002. Conociendo nuestras escuelas: un acercamiento etnográfico a la cultura escolar. Barcelona: Paidós.

CONSEJO NACIONAL DE FOMENTO EDUCATIVO. 2014. Programa Institucional del Consejo Nacional de Fomento Educativo 2014ح2018. 20/05/2014. México: DOF.

CUEVAS, Ana; HERNÁNDEZ, Roberto; LEAL, Brenda; MENDOZA, Christian. 2016. "Enseñanza-aprendizaje de ciencia e investigación en educación básica en México”. Revista Electrónica de Investigación Educativa, v. 18, n. 3.

ESQUIVEL, Juan Manuel. 2009. “Evaluación de los aprendizajes en el aula: una conceptualización renovada”. En: MARÍN, Elena; MARTÍNEZ, Felipe (Coords.). Avances y desafíos en la evaluación educativa. España: OEI/Fundación Santillana. p. 127-144.

GARCÍA, Adán; AGUILERA, María Antonieta; PÉREZ, María Guadalupe; MUÑOZ Gustavo. 2011. Evaluación de los aprendizajes en el aula: opiniones y prácticas de docentes de primaria en México. México: INEE.

GIMENO, José y PÉREZ, Ángel. 1996. Comprender y transformar la enseñanza. España: Ediciones Morata, Quinta Edición.

INSTITUTO NACIONAL PARA LA EVALUACIÓN DE LA EDUCACIÓN (2006). Los temas de la evaluación: Primarias comunitarias. México: INEE. 
LÓPEZ, Blanca; HINOJÓSA, Elsa. 2018. Evaluación del aprendizaje: alternativas y nuevos desarrollos. México: Editorial Trillas.

MARTÍNEZ, Guadalupe Iván; GUEVARA, Albertico. 2015. "La evaluación del desempeño docente". Revista Ra Ximhai, v. 4, n. 11, p. 113-124.

MARTÍNEZ RIZO, Felipe. 2008. "La evaluación de aprendizajes en América Latina”. Cuadernos de investigación, $\mathrm{n}$. 32. Resultados de investigación, INEE.

MARTÍNEZ RIZO, Felipe. 2013. "Dificultades para implementar la evaluación formativa: Revisión de literatura”. Perfiles educativos, México, IISUE-UNAM, v. 35, n. 139.

MONZÓN, Martha. 2015. "Evaluación del aprendizaje: un recorrido histórico y epistemológico" Academicus - Revista de Ciencias de la Educación, v. 6, n. 1, p. 12-24.

MORENO, Tiburcio. 2011. "La cultura de la evaluación y la mejora de la escuela”. Perfiles educativos, México, IISUE-UNAM, v. 33, n. 131, p. 116-130.

ROMÁN, Marcela. 2003. “¿Por qué los docentes no pueden desarrollar procesos de enseñanza-aprendizaje de calidad en contextos sociales vulnerables?”. Revista Persona y Sociedad, v. 1, n. 17, p. 113-128.

SECRETARÍA DE EDUCACIÓN PÚBLICA. 2013a. Programa Sectorial de Educación 2013-2018. Ciudad de México: Secretaría de Educación Pública. Disponible en: http: / /www.sep.gob.mx/es/ sep1/programa_sectorial_de_educacion_13_18. Acceso en: 16 de enero de 2019.

SECRETARÍA DE EDUCACIÓN PÚBLICA. 2013b. Censo de Escuelas, Maestros y Alumnos de Educación Básico y Especial. Ciudad de México: Secretaría de Educación Pública. 
Resumen: Se estudia el proceso de enseñanza-aprendizaje y su evaluación en escuelas indígenas y comunitarias situadas en ámbitos rurales del sureste mexicano con el objetivo de determinar la manera en que se ajustan a las necesidades de los educandos y si impactan en su aprendizaje. Mediante una combinación de técnicas predominantemente cualitativas - entrevistas abiertas, observación participante, grupos focales y una encuesta puestas en juego entre marzo y agosto de 2014 en zonas rurales aledañas a las principales ciudades de los estados de Quintana Roo, Campeche, Yucatán, Chiapas y Tabasco, se captan las prácticas vinculadas a la evaluación docente y las percepciones y discursos de algunos de los actores intervinientes de la comunidad educativa (estudiantes, docentes y funcionarios). Los resultados muestran que en los procesos de enseñanza-aprendizaje y en las prácticas áulicas se tiende a homologar las capacidades de niños y jóvenes. A pesar de los esfuerzos por lograr que sus alumnos asimilen la información proporcionada, los docentes cuentan con escasa formación pedagógica y con pocas herramientas de evaluación, lo que redunda en evaluaciones que no llegan a atender los contextos específicos de las realidades que enfrentan estas modalidades educativas.

Palabras-clave: evaluación docente; educación comunitaria; derecho a la educación; contextos rurales.
Abstract: The article focuses on the teaching-learning process and its evaluation in indigenous and community schools located in rural areas of southeastern Mexico with the objective of determining how they adjust to the needs of the learners and the reach of their impact on their learning processes. Through a combination of predominantly qualitative techniques - open interviews, participant observation, focus groups and a survey carried out between March and August 2014 in rural areas near the main cities of the states of Quintana Roo, Campeche, Yucatán, Chiapas and Tabasco, we recorded practices linked to teacher evaluation and the perceptions and speeches of some of the actors involved in the educational community (students, teachers and civil servants). The results show that the teaching-learning processes and classroom practices tend to standardize the capacities of children and young people. Despite their efforts to get students to assimilate the information provided, teachers have little pedagogical training and few evaluation tools, which results in evaluations that do not address the specific contexts of the realities faced by these educational modalities.

Keywords: teacher evaluation; community education; right to education; rural contexts. 